\title{
Dental Treatments in Sports Dentistry - Oral Care of Athlete, Mouthguards and Dental Trauma Management
}

\section{Sneha Divekar*}

Sports Dentist, Faculty of Certificate in Sports Dentistry Course, ISST, Clinic

Director - Dental Fitness and Polyclinic, Cosmetic Dentistry and Sports Dentistry

Centre, Pune, India

*Corresponding Author: Sneha Divekar, Sports Dentist, Faculty of Certificate in Sports Dentistry Course, ISST, Clinic Director - Dental Fitness and Polyclinic, Cosmetic Dentistry and Sports Dentistry Centre, Pune, India.
Received: July 29, 2021

Published: September 18, 2021

(C) All rights are reserved by Sneha Divekar.

\section{Abstract}

As more contact sports are played, the chances of dental injuries in sports are increasing by the day. That is why, it is important that the dentist insists that the athletes, esp. those playing contact need to wear mouthguards. But as awareness about mouthguards is not enough, dental trauma is often seen in sports that need to be treated. Also, along with dental injuries management, athletes need good dental care and oral hygiene maintenance which they quite often tend to neglect. That is why, sports dentistry as a new and upcoming topic in dentistry needs to be introduced to dentists. The dentists need to know what are the various types of dental treatments that the dentist will require to perform. Getting trained as a sports dentist and attaching as a consultant sports dentist to sports clubs, academies, schools, colleges, etc will be beneficial not only for the athletes but for the dentists as well.

Keywords: Sports Dentistry; Dental Care of Athletes; Mouthguards; Oral Hygiene; Dental Trauma in Sports

\section{Introduction}

With the world waiting with immense enthusiasm for Tokyo Olympics to begin and looking at the large number of athletes participating and representing India across various sports, it can be safe to say that Sports in India today is emerging as an important component of socio-economic development of our country. The active participation in sports improves community health and productivity, reduces medical expenses, imbibes discipline in character and enhances social cohesion. The execution of a mega sporting event helps in developing infrastructure, generating employment, securing inflow of foreign capital and thus contributes significant- ly to the economic development of our country. Therefore, it can be said that the impact of sports on the society is multi-dimensional.

However, it is of growing concern about the injuries that occur during participation in organized sports. Injuries during sport appear to be unavoidable, and up to $30 \%$ to $40 \%$ of all accidents in children and adolescents occur during sports. The prevalence of sports injuries vary widely. Injuries in youth sports account for substantial morbidity and cost. Sports injuries result from acute trauma or repetitive stress associated with athletic activities. Dental injuries are the most common type of oro-facial injury sustained during participation in sports with the increased popularity of con- 
tact sports. Contact sports are defined as those sports in which players physically interact with each other, trying to prevent the opposing team or person from winning. There is seldom very high incidence of dental trauma compared with all accidents in contact sports varying from $2 \%$ to $33 \%$ [1]. Dental and soft tissue injuries are commonly associated with some Top Indian collision and contact sports such as football, hockey, boxing, cricket, soccer, bicycling, skating, gymnastics, basketball, volleyball, karate, kabaddi, etc.

Also, along with dental injuries management, to get an edge to improve their performance, our athletes need, above all, good dental care and oral hygiene maintenance which they quite often tend to neglect. Using high quality mouthguards for injury prevention will increase their confidence while playing. Hence, introduction of sports dentistry to both the dentists and the athlete health care team is equally essential. There are various aspects of Sports Dentistry and many dental treatments can be catered under sports dental care like general dental care of athletes, treatment of athlete specific issues like dental erosion, bruxism, high decay rate, particular gum problems, tobacco counselling, nutrition counselling for better oral health and of course mouthguards and dental trauma management.

\section{Dental treatments done by the sports dentist}

Gum issues of athletes, teeth cleaning and motivation for better oral hygiene

Gingivitis and periodontitis are two of the most common oral infections seen in athletes. Studies have revealed specific periodontal diseases that have a particularly high incidence amongst athletes. Three different periodontal consequences are seen: firstly, precocious alveolysis; secondly, gingival hypertrophy and finally, swimmers' calculus. Generally speaking, an individual becomes more vulnerable to periodontal disease if subject to bad oral hygiene and a diversity of risk factors including bacterial interactions and an impaired host - response system. Athletes may therefore be particularly susceptible if suffering from a weaker immune system linked to overtraining, coupled to an unfavourable oral environment. Another influential factor is undoubtedly that of hyposalivation associated to intensive, prolonged sporting effort, hence a diminution of the protective factors saliva provides the buccal cavity [2]. Due to all these reasons, it is necessary that the athletes undergoes a dental check-up before every gaming season and make sure he/she has a good oral health. Along with risk to gum health, athletes have high incidence of dental caries and dental erosion due to their food habits and eating patterns. That is why, it is very important to educate the athletes, coaches and the parents about good oral hygiene practices and motivate them by doing periodic check-ups and teeth cleaning.

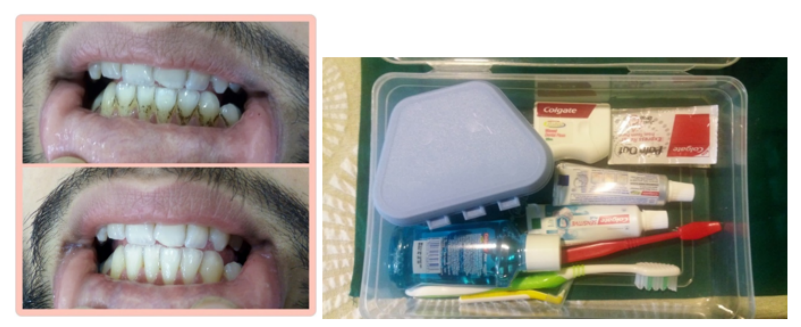

Figure 1: Teeth cleaning in athletes and motivation for oral hygiene (explain about oral care kit).

\section{Mouthguard fabrication}

A mouthguard is a protective device for the mouth that covers the teeth and gums to prevent and reduce injury to the teeth, arches, lips and gums. The 2 main types of mouthguards are boiland-bite mouthguards and custom-fabricated (not considering stock mouthguards as they are not recommended by dentists). The custom-fabricated mouthguard gives maximum protection to the mouth. The level of protection the athlete needs may change and depend on many factors. The protection from the mouthguard that the athlete wears depends upon the following main factors:

- $\quad$ The type of sport played

- $\quad$ Age and weight of the athlete

- Level of competition

- Level of impact that can take place during collision or injury.

The other factors include:

- Orthodontic bands

- $\quad$ Missing teeth

- Irregularities in bite or alignment of teeth

- Personal factors including medical or dental issues. 
That is why, when there are so many variations in the type of sport played, the type of impact, the age of the athlete, etc it makes sense to have a dental consultation and fabricate a customized mouthguard by a dentist for better protection and safety of the athlete [3]. The dentist will guide the athlete in the selection of any mouthguard; the ultimate decision has to be made by the athlete and in case of minors, by parents and the coach. Especially in a country like ours, such decisions are economically driven. So along with providing custom-fabricated mouthguards which are the best for athletes, the dentist also has to provide economical but high quality boil-and-bite mouthguards (for example, OPRO mouthguards).
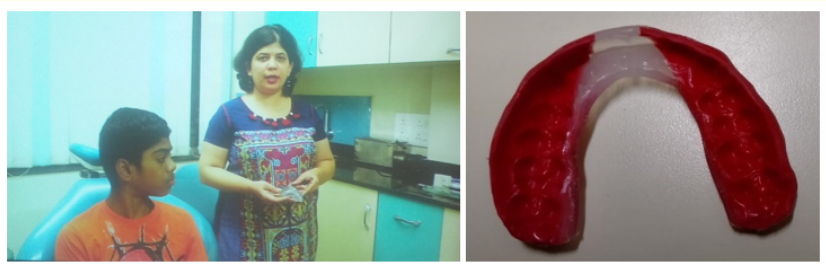

Figure 2: Customized mouthguard for 12yr boxing athlete.
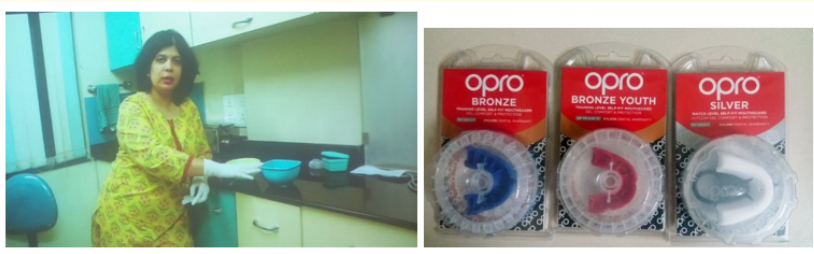

Figure 3: Making high quality boil-and-bite mouthguard for athletes.

\section{Dental trauma management of athletes}

Dental trauma management in athletes involves treatment of simple Elli's Class I fractures to treating crown-root fractures or dento-alveolar fractures. Jaw or oro-facial fractures will require the intervention of the oral and maxillo-facial surgeon. But the sports dentist on a routine basis may need to handle simple fracture cases which can be immediately corrected (that is within few days) after the injury. Composite restorations can be done in these cases to restore the teeth. The athlete has to be advised to wear a mouthguard in future to prevent further fracture of the restoration or the tooth. Presenting here are 2 such cases of Elli's Class II fracture treated with Composites. In case of pulp involvement or root fracture, 2 - 3 IOPA have to be taken to confirm the fracture lines. A pedodontist or endodontist needs to be consulted as the case maybe. The dentist will need to splint the teeth for 3 - 4 weeks, keep a follow-up with the athlete and do the root canal therapy later. In case of more loss of tooth structure, post-core needs to be done before placing the crown.

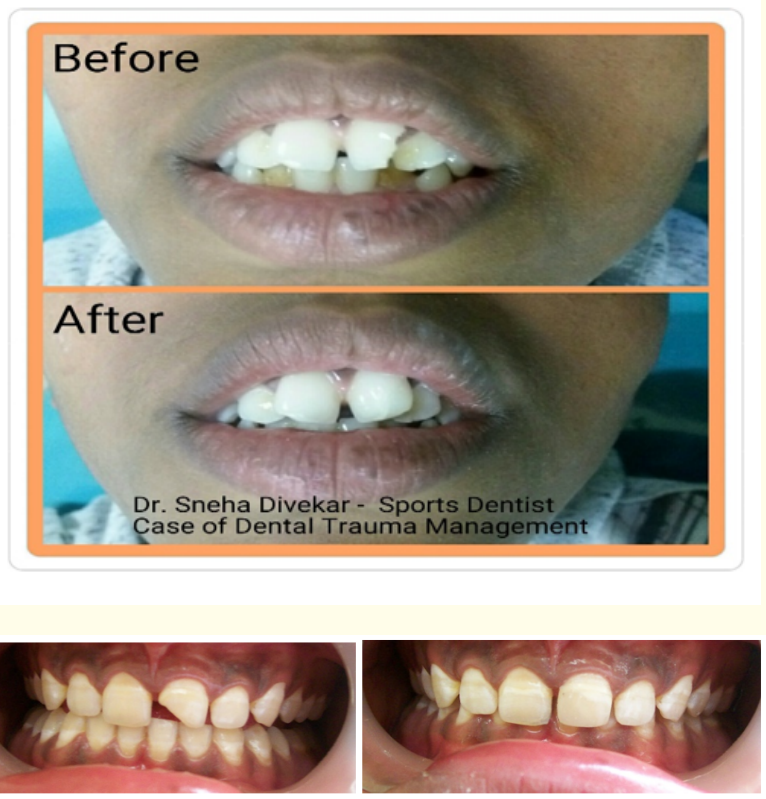

Figure 4: Patient 1 and 2: Cases of Class II fractures treated with composite restorations.

\section{Conclusion}

Sports Dentistry is a well-known subject in countries like USA, UK, Japan, etc. India too needs a systematic approach for how the dental profession can interact with the sports world, including opportunities for dentists in athletics. There is a great need for "Team Dentist" from high schools to professional teams. The dentists can assist athletes and incorporate knowledge into their private prac- 
tices in three main ways, i.e. by conducting pre-season oral health screenings, fabricating custom-made mouthguards, and being available for emergency care. Dentists can educate the general public, parents/guardians, staffs of emergency department, coaches and athletes regarding health risks and benefits of mouth guards including the importance of emergency care for orofacial injuries. It is advisable for the sporting community to have the sports dentistry centres all over the various cities across India identified for a quick approach when needed.

Note: For all those who are interested in increasing their knowledge about Sports Dentistry, we have a course called "Certificate in Sports Dentistry". Dr Sneha Divekar is the course faculty and the course is organized by Institute of Sports Science and Technology, Pune. It is a 3 months distance learning course with a 1 day contact workshop. For more details, contact Dr Sneha on 9822910294.

\section{Bibliography}

1. Neha Sharma., et al. "Sports Dentistry - It's time to change your game".

2. Siobhan $\mathrm{C}$ Budd and Jean-Christophe Egea. "Sport and Oral Health - A Concise Guide".

3. Julian Hodges. Mouthguards and Sports Safety (A Unique, OneStop, Practical, No-Nonsense Resource for Everyone Who Recommends or Should Wear a Mouthguard).

\section{Volume 5 Issue 10 October 2021} (C) All rights are reserved by Sneha Divekar. 